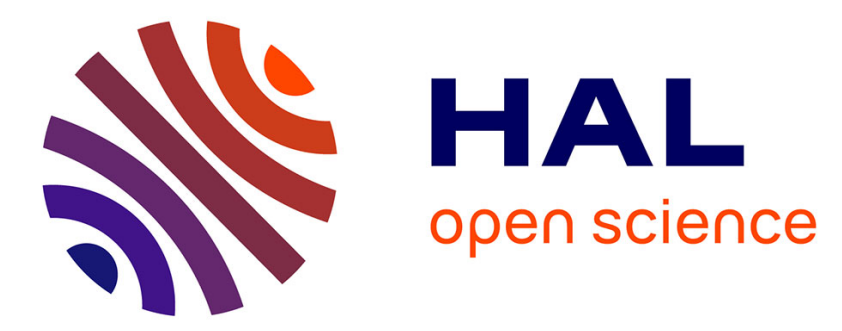

\title{
RANS models validation of the flow separation on a $6: 1$ prolate spheroid at angle of incidence
}

Miguel Torrente Pardo, Caroline Lienard, Ronan Boisard, Damien Desvigne, Michel Costes

\section{- To cite this version:}

Miguel Torrente Pardo, Caroline Lienard, Ronan Boisard, Damien Desvigne, Michel Costes. RANS models validation of the flow separation on a 6:1 prolate spheroid at angle of incidence. AIAA Scitech 2020 Forum (Session: Computational Fluid Dynamics Applications II), Jan 2020, Orlando, United States. 10.2514/6.2020-2237 . hal-03215932

\section{HAL Id: hal-03215932 \\ https://hal.science/hal-03215932}

Submitted on 3 May 2021

HAL is a multi-disciplinary open access archive for the deposit and dissemination of scientific research documents, whether they are published or not. The documents may come from teaching and research institutions in France or abroad, or from public or private research centers.
L'archive ouverte pluridisciplinaire HAL, est destinée au dépôt et à la diffusion de documents scientifiques de niveau recherche, publiés ou non, émanant des établissements d'enseignement et de recherche français ou étrangers, des laboratoires publics ou privés. 


\title{
RANS models validation of the flow separation on a 6:1 prolate spheroid at angle of incidence
}

\author{
Miguel Torrente Pardo*1,2 Caroline Lienard $^{\dagger 2}$, Ronan Boisard ${ }^{\ddagger 2}$, Damien Desvigne ${ }^{\S 1}$, and Michel Costes ${ }^{\mathrm{I} / 2}$ \\ ${ }^{1}$ Airbus Helicopters S.A.S.,Marseille Provence International Airport, F-13725 Marignane cedex, France \\ ${ }^{2}$ ONERA, 8 rue des Vertugadins, F-92190 Meudon, France
}

The prediction of the flow separation on helicopters might be quite complex by computational or experimental means, as several phenomena will emerge and interact amongst themselves. A simplification of the geometry can be done in order to isolate the phenomenon of interest. The current paper investigates flow separation around a 6:1 prolate spheroid at incidence, as this academic test case is a good simplification of a helicopter's engine cowling. A performance assessment of different turbulence models using a RANS approach is done in order to develop a methodology for studying separation. Comparing results with experimental measurements shows a rather good agreement using an Explicit Algebraic Reynolds Stress Model (EARSM) and a Differential Reynolds Stress Model (DRSM). On the other hand, the EARSM model seems to offer the best compromise between results accuracy and convergence rate.

\section{Nomenclature}

$\begin{array}{ll}C_{f} & =\text { Friction Coefficient } \\ C_{p} & =\text { Pressure Coefficient } \\ R e_{L} & =\text { Spheroid Length based Reynolds number } \\ u_{e} & =\text { Freestream Velocity beyond the boundary layer } \\ U_{\infty} & =\text { Freestream Velocity } \\ y^{+} & =\text {Dimensionless Wall Distance } \\ \tau_{w} & =\text { Wall Friction } \\ \text { CFD } & =\text { Computational Fluid Dynamics } \\ \text { DRSM } & =\text { Differential Reynolds Stress Model } \\ \text { EARSM } & =\text { Explicit Algebraic Reynolds Stress Model } \\ \text { LDV } & =\text { Laser Doppler Velocimetry } \\ \text { LES } & =\text { Large Eddy Simulations } \\ \text { QCR } & =\text { Quadratic Constitutive Relation } \\ \text { RANS } & =\text { Reynolds Averaged Navier Stokes } \\ \text { SA } & =\text { Spalart Allmaras } \\ \text { SST } & =\text { Shear Stress Tensor } \\ \text { URANS } & =\text { Unsteady Reynolds Averaged Navier Stokes }\end{array}$

\section{Introduction}

Nowadays, an overall better understanding of helicopter aerodynamics is obtained by combining numerical simulations, wind tunnel and flight tests. Nevertheless, transient and unsteady flows remain challenging to predict, owing to their complex and three-dimensional behaviour, such as flow separation and wake convection. Both of them are phenomena hard to anticipate which can have destabilizing effects on the forces and moments balance of the helicopter. Indeed, their combination is involved in critical phenomena regarding the helicopter handling such as Tail-Shake (Roesch

\footnotetext{
*PhD. Student, External Aerodynamics Department, miguel.torrente-pardo@airbus.com

${ }^{\dagger}$ Research Scientist, Aerodynamics, Aeroelasticity and Acoustics Department, caroline.lienard@onera.fr

Research Scientist, Aerodynamics, Aeroelasticity and Acoustics Department, ronan.boisard@onera.fr

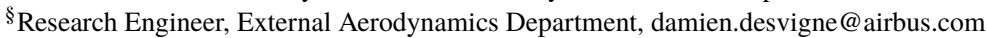

IResearch Engineer, Aerodynamics, Aeroelasticity and Acoustics Department, michel.costes@onera.fr
} 
\& Dequin [1], Mazzucchelli \& Willson [2]). Therefore, improving numerical simulations can provide aerodynamically de-risked designs at an earlier stage of the conception, avoiding wind tunnels' scale effects and flight tests' high costs.

In order to overcome the difficulty of simulating the aerodynamics of the whole helicopter, this study focuses on a single aerodynamic phenomenon as if only one part of the helicopter was considered. In this way, the issue of interest is isolated, and a more detailed and accurate analysis study can be devoted to it. This study aims at understanding the physics of separation -and more specifically crossflow separation- as well as simulating it in order to investigate the aerodynamic phenomena related to it. Indeed, capturing correctly flow separation is essential in order to be able to later capture the effect of turbulent wake convection on the tail surfaces of the helicopter.

In the literature, most of crossflow separation cases are studied from bodies of revolution, and the 6:1 prolate spheroid at angle of incidence is largely the most investigated case by experimental and computational means (Figure 1 1 ). Even if the geometry of the spheroid can seem simple, several complex aerodynamic phenomena appear. As seen in Figure 1 , the spheroid has a great lateral surface curvature with respect to the longitudinal one; indeed, the crossflow separation is generated by this characteristic, that produces a curvature of the streamlines. Then, this crossflow separation generates several pairs of counter-rotating vortices, depending on the incidence, that are convected downstream. The interest of this test case is its similarity to the geometries of some parts of the helicopters, notably engine cowlings as seen in Figure 1p), as they will trigger the same phenomena.
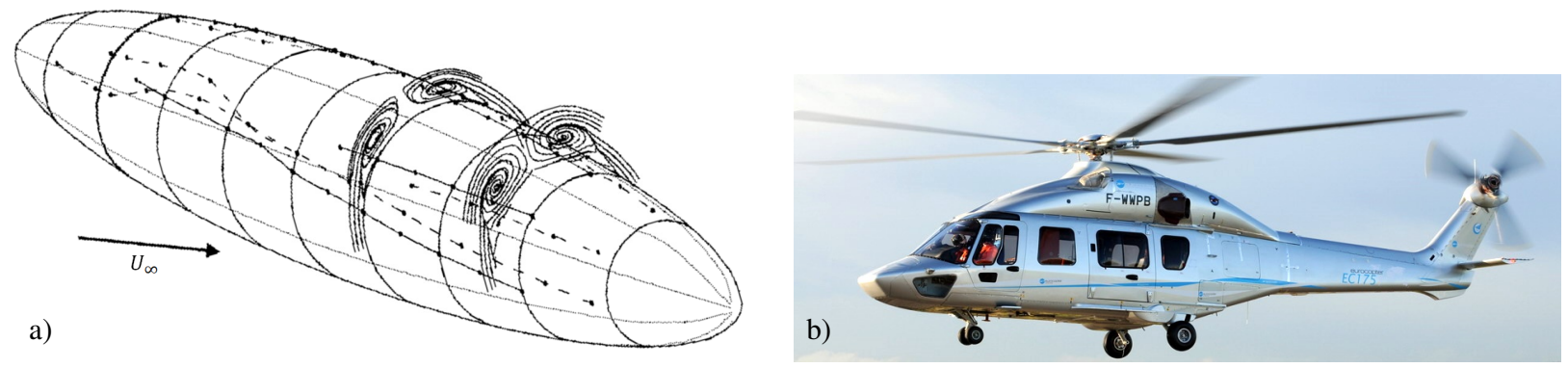

Fig. 1 The 6:1 prolate spheroid geometry: a) Overview of spheroid flow by Wetzel et al. [3], continuous lines are the oil flow separation locations, whilst dashed lines are the friction minima ; b) Typical helicopter engine cowling (Airbus Helicopters H175 prototype).

The present work refers to the experimental data of the Virginia Polytechnic Institute campaigns during the 1990's. In these campaigns, the $6: 1$ prolate spheroid was tested at $R e_{L}=4.2 \times 10^{6}$ at several incidence angles and with a transition band at $20 \%$ length from the nose to set the position of the laminar-to-turbulent transition. Ahn \& Simpson [4], and Chesnakas \& Simpson [5] and [6] characterized the flow separation by surface pressure measurements and Laser Doppler Velocimetry (LDV) measurements of the velocities in the boundary layer. They correlated these measurements to the crossflow separation location. In addition, Ahn \& Simpson [4] detected that for Reynolds numbers higher than $2.5 \times 10^{6}$ (critical Reynolds number), the transition trip had negligeable influence on the separation lines position. Wetzel [7] departed from previous studies to later investigate the unsteady behavior of the aerodynamics for the spheroid following a turning maneuver, in order to study the influence into flow separation. Goody et al. [8] analyzed the pressure fluctuations in the boundary layer of the spheroid at several angles of attack, obtaining relatively weak values. Moreover, Wetzel et al. [3] studied the physics of crossflow separation and achieved an overview of the accuracy of the different crossflow criteria. They showed the requirement of a global method to correctly calculate this kind of separation. In the present study, all the results of these tests are used with the intention of validating the different Computational Fluid Dynamics (CFD) models and their accuracy for capturing crossflow separation.

From the beginning of the 2000's, several investigations have used this database to test several computational models' performance. These investigations started studying the test case using the Reynolds-Averaged Navier-Stokes (RANS) equations. For instance, Tsai \& Whitney [9] used the $k-\epsilon$ turbulence model with a wall function for simulating the crossflow. The flow characteristics were captured qualitatively well but some differences for the friction coefficient are obtained. Thus, wall constants had to be modified in order to improve results. A similar research was done by Rhee \& Hino [10]: the Spalart-Allamaras turbulence model was used for predicting the separated flow. This model succeeded in capturing the primary pair of vortices characteristics but the secondary one was not well predicted when compared to experimental data. Later studies simulated the spheroid flow using more advanced techniques such as Unsteady RANS (URANS), Large Eddy Simulations (LES) and hybrid RANS-LES, taking advantage of the computing power improvement. Wikström et al. [11], Constantinescu et al. [12], Fureby et al. [13] and Xiao et al. [14] used 
hybrid RANS-LES, usually obtaining good overall behaviors of the separated flow, even if differences still existed when calculating the secondary separation for the first studies. These simulations did not present any unsteadiness. On the other hand, Mor-Yossef [15] used different DRSM turbulence models for comparing calculations of the velocity and the Reynolds stress profiles in the boundary layer. The SSG/LRR- $\omega$ model provided very good agreement with experimental data for the velocity profiles and for the normal Reynolds stress tensor, but the shear stress were neither captured with this model nor the others DRSM ones. Recently, Fureby \& Norrison [16] compared RANS simulations (k- $\omega$ Menter SST), hybrid RANS-LES (IDDES) using a RANS grid and a LES grid, and LES simulations (LDKM with and without a trip model) with the experimental data. RANS and LES approachs showed well-correlated results when studying the mean and rms axial velocity, contrarily to the IDDES simulations. Tripped and untripped LES calculations succeeded in capturing the unsteady behaviour of the counter-rotating vortices; the only difference between them was the position of the vortices, as the tripped case shifted them upward. These studies highlighted that only LES simulations and hybrid models using LES grids can detect the weak unsteady behaviour, as this unsteadiness is originated in the boundary layer. On the other hand, Mor-Yossef [15] and Fureby \& Norrison [16] showed that RANS calculations allow to capture the mean aerodynamic characteristics for the $6: 1$ prolate spheroid test case in a satisfactory way. In effect, the improvement of computational technologies permits to run RANS calculations with finer grids than in the 2000's, conserving acceptable calculation times for industrial purposes.

In this context, this investigation aims to understand and characterize the physics of crossflow separation using different RANS models within the same grid. In this way, a comparison between different turbulence models and with the experimental data is done in order to determine the most appropriate one for capturing flow separation. Amongst all the turbulence models which have been evaluated, Spalart-Allmaras, k- $\omega$ Menter SST, the k- $\omega$ Explicit Algebraic Reynolds Stress Model (EARSM) from Hellsten, and the SSG-LRR- $\omega$ Differential Reynolds Stress Model (DRSM) will be presented in the current paper. From the conclusions of this comparison, the best models are selected and will be applied in a future helicopter test case.

\section{The 6:1 Prolate Spheroid Test Case}

The database obtained by the wind tunnel experimental campaigns led by R.L. Simpson from the Virginia Polytechnic Institute is used for validating the computed results obtained by the simulations done in this study. This campaign used a prolate spheroid with 6 to 1 aspect ratio, where its length is $1.347 \mathrm{~m}$, and its diameter at the center is $0.229 \mathrm{~m}$ (Figure 22. The wind tunnel used for the campaign was the State University Stability Wind Tunnel, that is a continuous, closed return, subsonic wind tunnel with a length of $7 \mathrm{~m}$ and a square test section of $1.8 \times 1.8-\mathrm{m}^{2}$. This gives a blockage ratio of $2.3 \%$. The wind tunnel provided a low freestream turbulence level of the order of $0.03 \%$. In the experimental tests, transition on the spheroid leading edge is triggered with a circumferential trip wire located at $20 \%$ length from the spheroid nose. This ensures the laminar-to-turbulent transition position and then a turbulent regime around the spheroid. Wind tunnel tests were done at a length based Reynolds number of $R e_{L}=4.2 \times 10^{6}$. Two angles of attack have been studied during the experimental campaign of interest: $10^{\circ}$ and $20^{\circ}$ and each case has its own flow characteristics. Indeed, the $10^{\circ}$ test case has a single pair of vortices whilst the larger incidence of the $20^{\circ}$ test case generates a pair of secondary and counter-rotating vortices inside the primary one (see Figure 3). On the other hand, measurements have been focused on two longitudinal positions : $60.0 \%$ and $77.2 \%$ length from the spheroid nose.

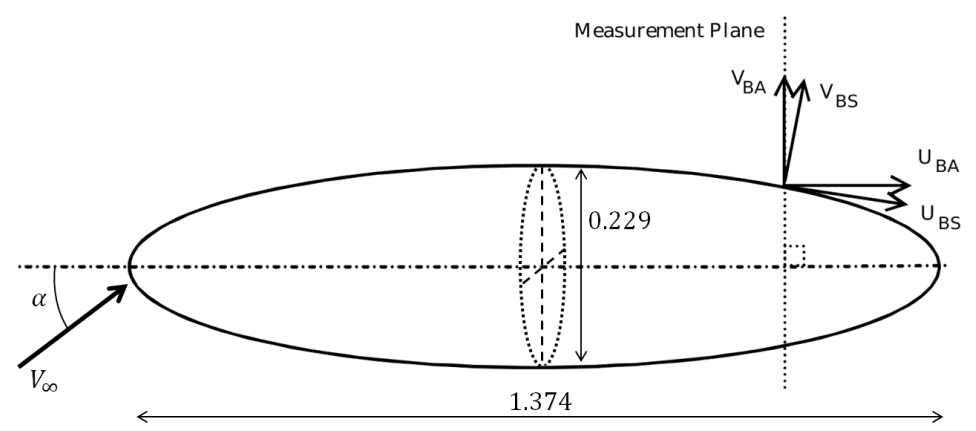

Fig. 2 6:1 prolate spheroid geometry from Wetzel et al. [3]. $\alpha$ is the angle of attack and $\mathbf{U}_{\infty}$ the airflow velocity. Coordinate systems in measurement plane, are Body Axis and the Body Surface. 
The flow characteristics measurements that are going to be used in the present work are: mean static skin-pressures obtained with pressure transducers, the skin-friction with LDV, velocity profiles and the Reynolds stress tensor in the boundary layer with LDV, as well as oil flow visualizations of the separation position. Velocity profiles shown later are given in a Body Surface coordinate system (Figure 2), with $U$ tangential to the surface and pointing to the tail of the model, $V$ perpendicular to the skin (positive outwards) and $W$ tangent to the surface and forming a right-handed coordinate system.

The influence of vortices on skin pressure and friction is large and should be understood before comparing results from experiments and simulations (Figure 3). Pressure maxima and minima are difficult to correlate with the vortices position due to the flow three-dimensionality, as seen in Figure 3 a) and b). As a matter of fact, the interaction between vortices and the body have a strong impact on wall pressure distribution. Pressure minima are normally found before flow separation, whilst maxima are found before the reattachment flow. On the other hand, azimuthal friction maxima and minima are easier to correlate with separation and reattachment. Figures 3 c) and d) show that separation points are close to friction minima and reattachment points are close to friction maxima. The $10^{\circ}$ test case has not been presented in Figure 3, but the azimuthal distributions at the two longitudinal positions are studied at the Results chapter, where they are extensively explained.

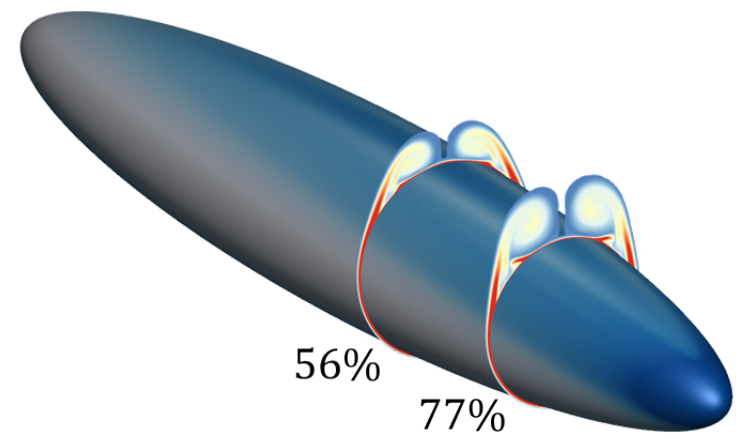

a)

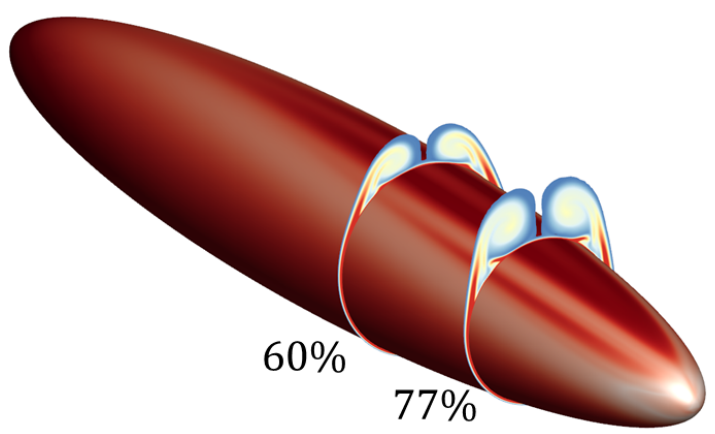

c)
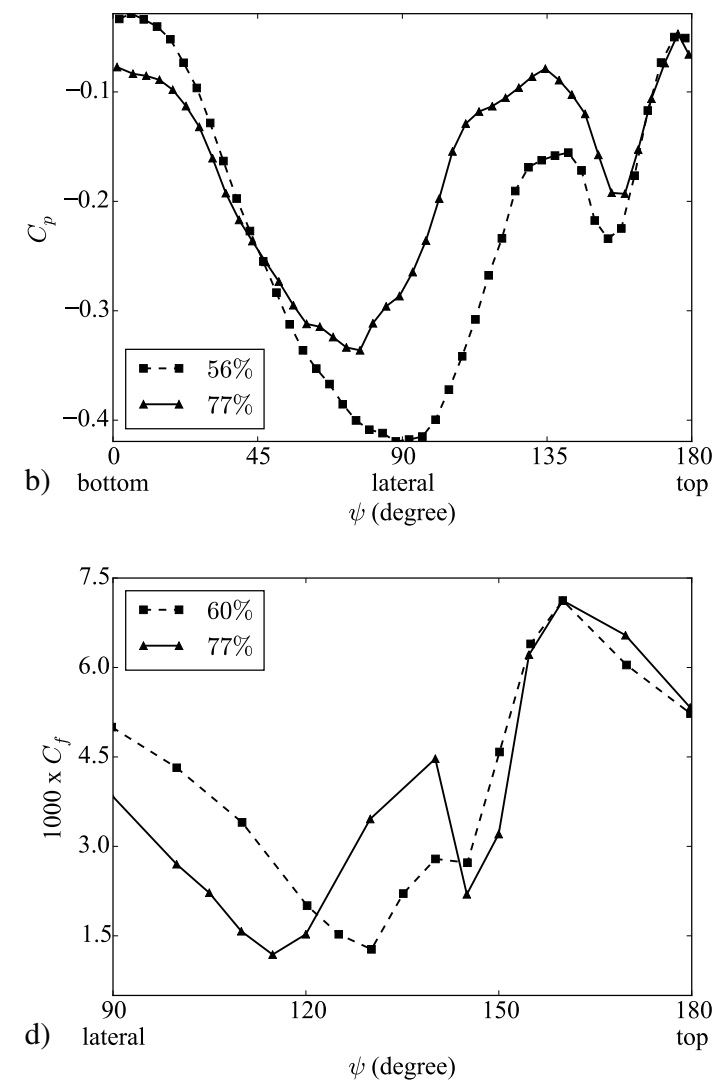

Fig. 3 Vortices influence on skin quantities at two longitudinal positions for the $20^{\circ}$ test case. Right figures a) and c) show EARSM Hellsten simulations results, with vorticity at two plans. Left figures b) and d) present the skin quantities azimuthal distributions. a) and b) present the skin pressure coefficient, whilst c) and d) present the friction coefficient obtained with the LDV measurements from Wetzel [7]. 


\section{CFD calculations}

\section{A. Numerical methods}

CFD calculations use the elsA multi-purpose software for aerodynamics, developed at ONERA and co-owned by ONERA, Airbus and Safran (Cambier et al. [17]). Compressible Navier-Stokes equations are solved using a finite-volume approach. The AUSM+(P) space discretization schema developed by Liou \& Edwards [18] is used for the inviscid part of the fluxes. In effect, this scheme is preferred to the Roe or Jameson ones for its lower numerical dissipation on the boundary layers and low-Mach capabilities. Viscous fluxes are discretized with a simple five points centred scheme. Convergence of the steady-state RANS equations is obtained with a backward Euler method and local time-stepping. The type of implicitation of the equation system used is a scalar LU-SSOR method.

The following turbulent models for closing the RANS equations are tested during this study: the one-equation Spalart-Allmaras model from Spalart \& Allmaras [19] (SA), the two-equation k- $\omega$ model with the SST correction of Menter [20] (Menter), the Kok extension of the two-equation k- $\omega$ model of Kok [21] (Kok), the two-equation k- $\omega$ model with the SST correction and a Quadratic Constitutive Relation proposed by Spalart [22] (MenterQCR), the Hellsten two-equation k- $\omega$ model from Hellsten [23] (EARSM), the version of the SSG- $\omega$ from Aupoix [24], and finally a blended SSG-LRR-omega RSM model from Cécora et al. [25]. The SA, Menter and Kok models use the Boussinesq's hypothesis, a linear dependence between the Reynolds stresses and the velocity gradient tensor. The MenterQCR model consists in adding a second nonlinear term to the linear Reynolds stress tensor calculated with the Boussinesq relation. The EARSM model is an extension of the two-equation k- $\omega$ model that modifies the Boussinesq's hypothesis for including the non-linear terms from the Reynolds stress transport. The SSG- $\omega$ and SSG-LRR- $\omega$ are DRSM models that model the transport of the six Reynolds stresses and the specific dissipation rate $\omega$.

The transition trip at 20\% length from the spheroid nose has not been considered in the simulations. Ahn \& Simpson [4] and Meier \& Kreplin [26] studied the influence of the transition trip location. Similar separation lines were found when the trip was placed at different locations and for Reynolds numbers above $4.2 \times 10^{6}$ critical one. Since velocity profiles before and after the different transition trips locations were similar, in the next experiments the transition trip was only used for stabilizing the transition and ensuring similar flow conditions downstream. For this reason, the lack of transition trip should have a minimal effect on the differences between experimental and computational results, as Mor-Yossef [15] shows. Additionally, Fureby et al. [16] compared LES simulations with and without a transition band, showing very little differences between the two simulations.

\section{B. Grid description}

The computational domain consists in a volume of $7-\mathrm{m}$ long and a $1.8 \times 1.8-\mathrm{m}^{2}$ section, with farfield conditions at the boundaries of the domain. This characteristic does not allow to consider the same blockage ratio as during experimental tests, and can impact the skin-pressure distribution. In addition, the experimental apparatus (sting-strut model support system) has never been considered, but this characteristic should have low-impact on the results.

The grid considered during this study has 30 million cells, with at least 30 cells in the boundary layer. The average wall distance $y^{+}$is equal to 0.8 and the aspect ratio in the boundary layer is lower than $5 \%$. In the near field (field one diameter far from the spheroid surface), cells of 5 to $7 \mathrm{~mm}$ can be found. Figure 4 shows a zoom of the surface trailing edge and field grid used for the numerical simulations.

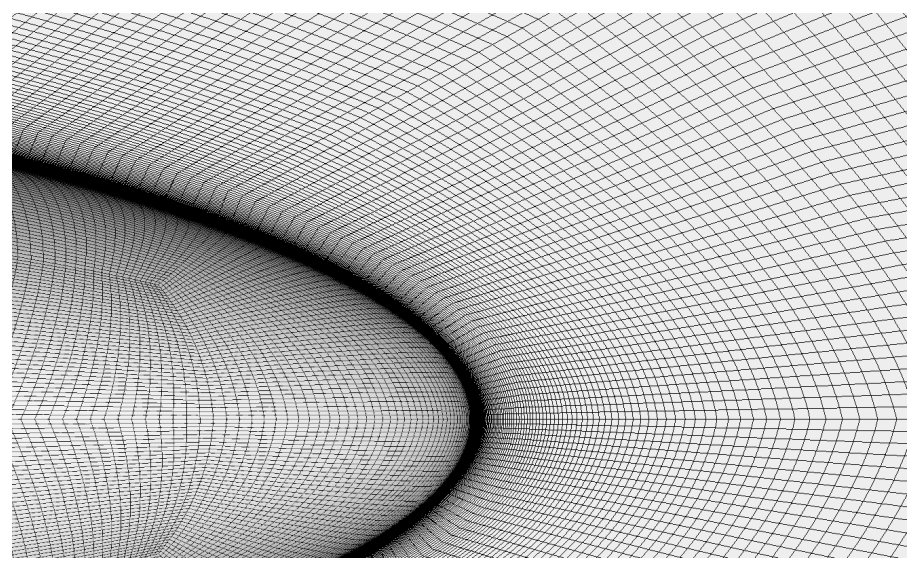

Fig. 4 Side view of the computational domain, zoom into the trailing edge. Flow goes from left to the right. 


\section{Determination of cross-flow separation location}

Flow separation is one of the most challenging topics in numerical aerodynamics due to its strongly three-dimensional character. Classically, two dimensional separations have been considered in order to reduce the complexity and simplify the problem. In this way, separation mechanisms can be well understood, numerical simulations can be tested and a criterion can be developed. However, real separations present much richer and complex physics than 2D ones. The study of the wall flow is very useful in order to understand flow topology. Indeed, several authors use the critical points theory for it, such as Delery [27]: flow separation and reattachment can be studied using this theory as seen in Figure 5 In the flow separation case, flow tends to get out of the body surface and thus, applying the continuity equation, friction lines tend to get closer among them, and close to a virtual line called the separation line. As a matter of fact, flow separation results in a vorticity expulsion from the body surface. On the other hand, reattachment will have an opposite behaviour: flow lines tend to dive into the body surface, and thus the friction lines will tend to move away from a virtual line called the reattachment line. In this case, the vorticity present in the separated flow region gets back into the boundary layer close to the wall.

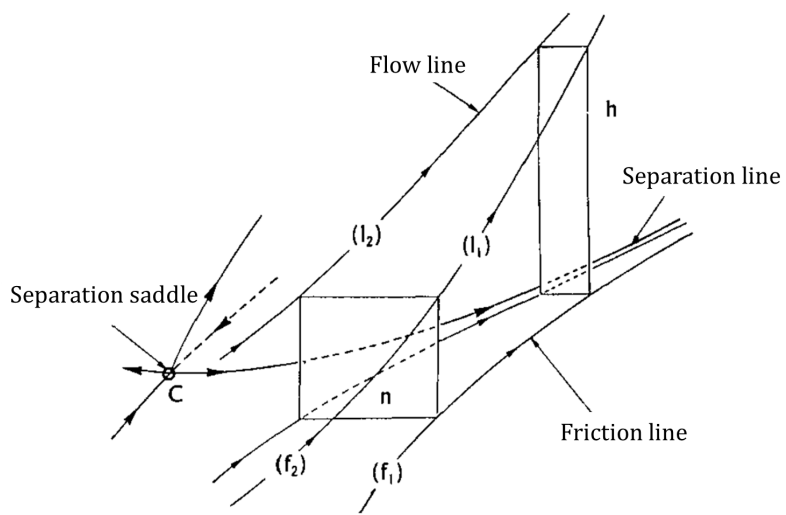

a)

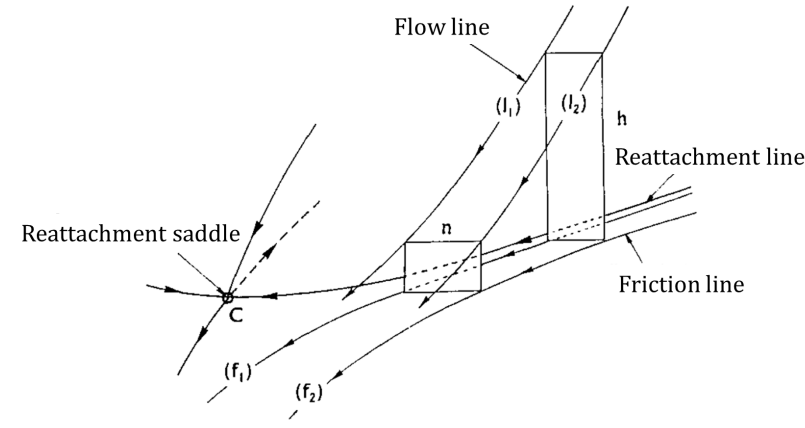

b)

Fig. 5 Overview of a) separation and b) reattachment using the critical points theory, from Delery [27].

The critical points theory establishes a relationship between nodes, focii points and saddle points. Nodes and focii are topologically similar: they are sources or sinks for the friction lines that are born from or disappear into streamlines in the flow field. On a body surface there are always at least two nodes (or focii): an attachment point and a separation one. Friction lines can only be originated from one attachment points whilst they have to end necessarily at a separation point. On the other hand, if two attachment points coexist in a body, a separation line is required to "separate" friction lines coming from these nodes. In this way, a separation line implies the existence of a saddle point. With this basis, a relationship is established between the amount of critical points: $\sum(n+f)-\sum s=2$, where $n$ are the nodes, $f$ are the focii and $s$ the saddle points.

However, cross-flow separation is a particular kind of separation, due to its non-unique character and its weak interaction with the local flow-field at the beginning of separation, as explained by Wetzel et al. [3] or Han \& Patel [28]. On one hand, the non-unique character of crossflow separation is because it does not originate from a unique singularity point or line. On the other hand, at the start of the crossflow separation, flow is nearly attached, and thus, it does not significantly interact with the freestream. In this way, it has a weak effect on the local flowfield. Spheroid flow and thus, crossflow separation can be studied using the critical points theory mentioned before. All the spheroid critical points are located at the trailing edge of the spheroid, excepting the leading edge attachment node, and they are shown in Figure 6 for the EARSM simulation as the number and location of predicted critical points depends on the turbulence model used. Trailing edge critical points are due to the influence of the crossflow vortices and the pressure gradient separation. This makes a total amount of 3 nodes, 4 focii and 5 saddle points, and thus the critical points relationship is checked. No critical points are found at the origin or vicinity of the crossflow separation lines, supporting the non-uniqueness hypothesis and weak interaction at the start of crossflow separation. Considering all these aspects, cross-flow separation can not be determined using the critical points theory. Additionally, this supports the fact that flow is always attached along the spheroid and that crossflow separation can rather be viewed as a boundary layer rolling around itself. 


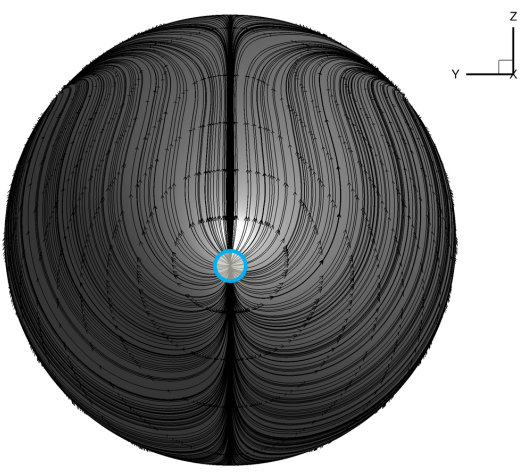

a)

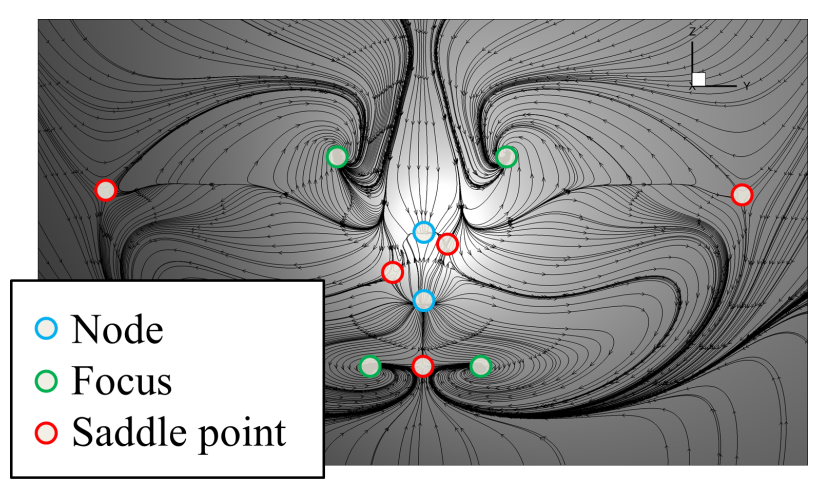

b)

Fig. 6 Skin flow using friction lines at the a) nose and b) the trailing edge of the 6:1 spheroid. Critical points are indicated with different coloured spots.

Therefore, a criterion is needed to determine the topological feature that behaves as a distinct separation line. Several criteria with a higher or lower accuracy have been developed and are largely presented by Wetzel et al. [3]. Criteria found are the minimum of friction, the detection of gradients in the surface pressure, or the maximum wall-normal velocity near separation, among others. That study shows the need of a global criterion in order to establish the crossflow separation line, which means a criterion non modified by the coordinate system used. Thus, most of the criteria exposed need to know a priori the position of the separation line to be able to calculate it. For example, one cannot find separation by examining secondary flow streamlines unless these streamlines are represented in a coordinate system that is oriented with the local separation line direction. On the other hand and contrary to what Wetzel et al. [3] state, friction minima does not seem to be an appropriate criterion: indeed Figure 1 a) shows that friction minima and oil visualisation lines are not coincident for few azimuthal degrees.

All these reasons hinder the calculation of the cross-separation line from simulations. Therefore, an hypothesis is needed to enable its calculation. Kreplin et al. [29] shows a simple criterion that considers separation where cross-friction is equal to zero, being cross-friction the friction component perpendicular to the body axis. This criterion assumes that the separation line is parallel to the spheroid direction. Kreplin et al. [29] showed a good agreement between this criterion and the oil visualizations for the spheroid at a Reynolds number of 6.5 millions and a $30^{\circ}$ angle of attack. In chapter IV.B, the good agreement between the oil visualizations and the cross-friction criterion is shown.

This crossflow separation criterion will have similarities with the longitudinal separation one (Figure 7 a). Indeed, longitudinal separation happens when friction becomes equal to zero $\tau_{w}=0$ : this means a change in the friction sign regarding to the freestream velocity $u_{e}$ and indicates the existence of reverse flow. Mathematically it is expressed as $\tau_{w} \cdot u_{e}=0$. On the other hand, when studying the crossflow separation, the change of sign is not observed in relation to the longitudinal flow but to the crossflow (Figure $7 \mathrm{~b}$ ).

a)

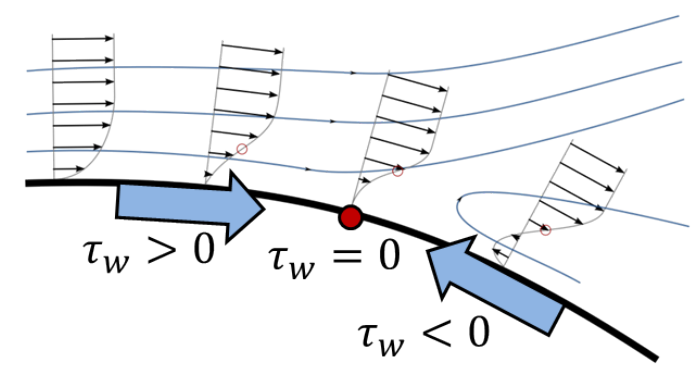

Fig. 7 Velocity profiles for different flow separations: a) Classical 2D separation (where $\tau_{w}$ is the wall friction); b) Longitudinal and crossflow velocity profiles for separated crossflow (from Chesnakas \& Simpson [6]). 
Cross-friction azimuthal evolution and crossflow velocity lines can be associated as Figure 8 shows. In the separation points, the friction lines converge towards the separation line as explained at the beginning of this chapter. In this way, the velocity lines will also converge and get ejected from the body surface. On the other hand, next to the reattachment points, friction lines will diverge, whereas streamlines will get close to the surface. The behaviour of streamlines is observed in Figure 8 b). In this figure, separation and reattachment are indicated with a $S$ and a $R$ respectively, while primary and secondary positions with the subindex 1 and 2 .

a)
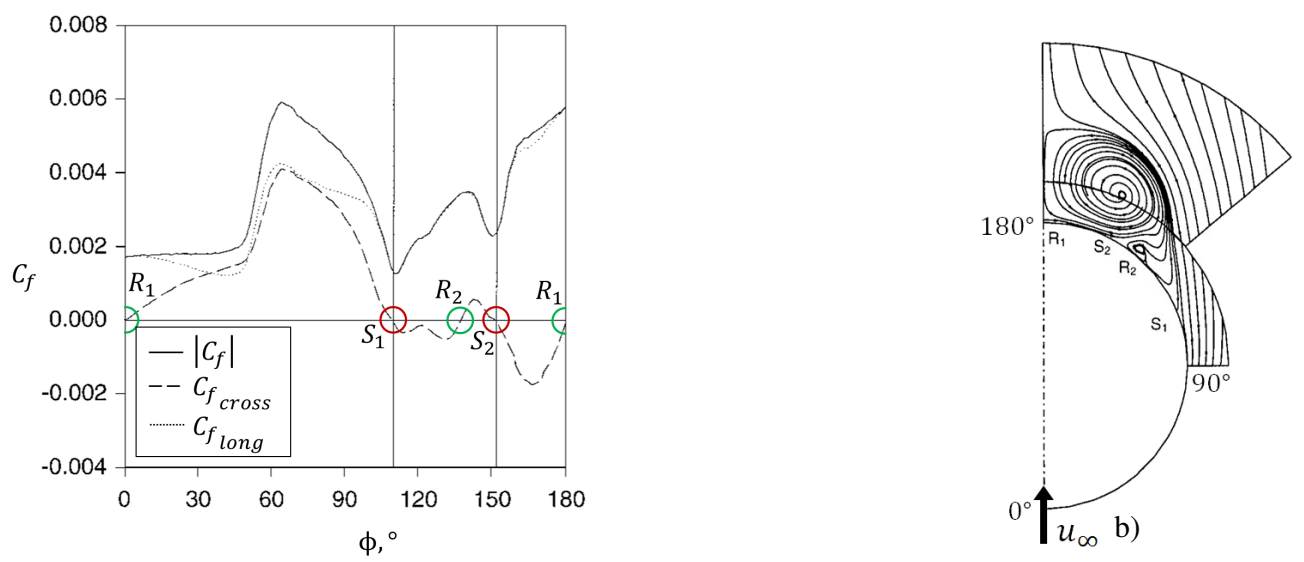

Fig. 8 Crossflow friction and velocity streamlineslines for two different test cases. a) $30^{\circ}$ incidence angle, section at 77\% from Kreplin et al. [29]; b) section at 77\% position from Chesnakas \& Simpson [5]

\section{Results}

\section{A. Comparison of model variants}

A large number of CFD simulations have been done during this study. First of all, in order to compare them in the clearest way, an initial comparison is established between similar models to rank them. This comparison concerns the $k-\omega$ models and Reynolds Stress Models. The $k-\omega$ models comprises $k-\omega$ Kok, $k-\omega$ Menter SST and $k-\omega$ Menter SST with the QCR closure. EARSM is not considered there as the relationships to calculate the Reynolds Stress are not based on the Boussinesq hypothesis, and thus they are different enough from other $k-\omega$ based models to consider it as a distinct model by itself. In the same way as with $k-\omega$ models, results from SSG- $\omega$ DRSM and SSG-LRR- $\omega$ DRSM are compared too. Even if both $10^{\circ}$ and $20^{\circ}$ incidences have been considered in the present comparison, only the $20^{\circ}$ test case is shown during this first part for legibility reasons.
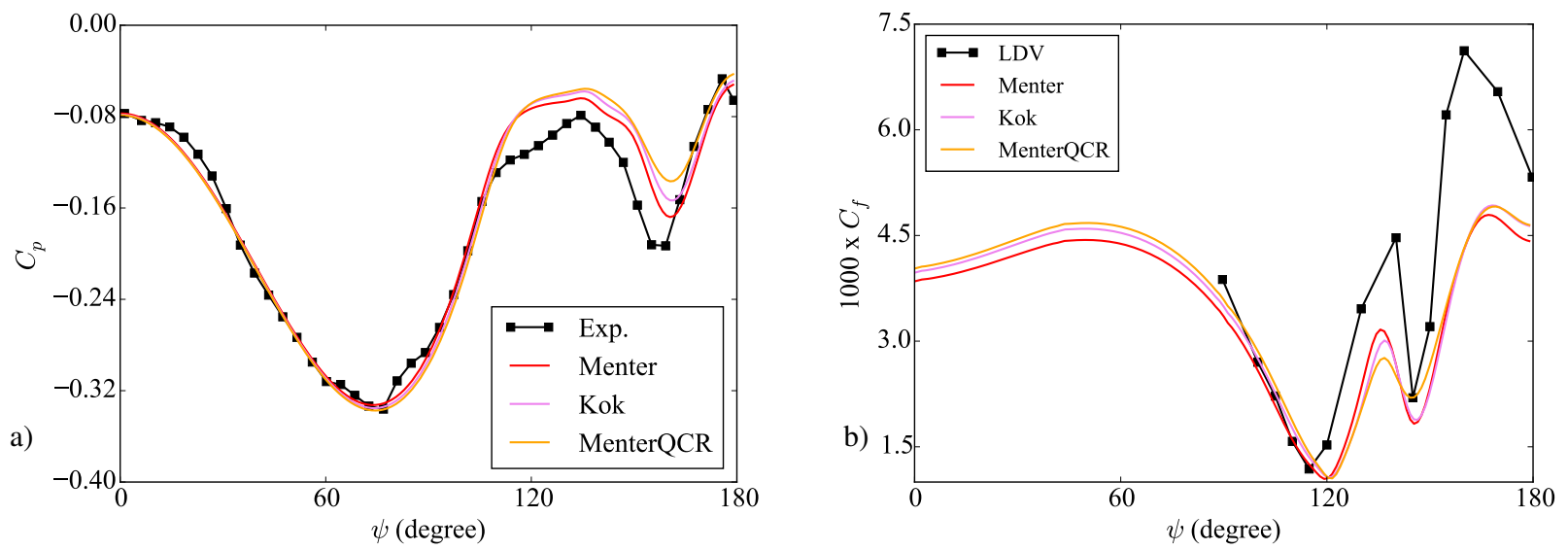

Fig. 9 Azimuthal distributions of $k-\omega$ models at a 77\% longitudinal slice $20^{\circ}$ of angle of attack. a) Skin pressure coefficient and $b$ ) friction coefficient azimuthal distributions. 
A first comparison between $k-\omega$ models is presented in Figure 9 This figure shows the skin pressure and friction azimuthal distributions at the $77 \%$ longitudinal position obtained with Kok, Menter and MenterQCR. Similar behaviours are shown by the three models, and the only differences are found near maxima and minima of the pressure and skin-friction azimuthal distributions. From these points, a better correlation with experiments is observed for the Menter simulations. Indeed, this model succeed in better capturing trends, as well as the minima and maxima of skin pressure and friction generated by the vortices. On the other hand, MenterQCR shows the less satisfactory results when compared to LDV measurements, especially near the position of the second vortex. This model was conceived for better simulating corner flows which are not encountered in the present test case. Furthermore the QCR closure negatively affects the capability of k- $\omega$ models to predict 6:1 spheroid flow phenomena.

Secondly, a comparison between the two DRSM models is performed for determining which is better adapted for capturing crossflow separation. Azimuthal distributions by SSG- $\omega$ and SSG-LRR- $\omega$ models are shown in Figure 10. Similar behaviours are found in both simulations as far as pressure distributions are concerned. However, the friction distribution shows large divergences around both vortices position, either for the $10^{\circ}$ or the $20^{\circ}$ test cases. These differences are more evident from $150^{\circ}$ to the top of the spheroid (the secondary vortex separation and primary reattachment). Globally, SSG- $\omega$ model seems to produce more friction than SSG-LRR- $\omega$, and this is especially interesting when studying the friction in the windward side (before $90^{\circ}$ ), and around the $160^{\circ}$ azimuth (maximum of friction caused by the primary vortex reattachment). In the windward side SSG- $\omega$ seems to better follow the experimental trend, and around the $160^{\circ}$ azimuth SSG- $\omega$ is closer to the maximum of friction. On the other hand, SSG-LRR- $\omega$ seems to better capture the friction minimum generated by the primary separation, around $120^{\circ}$ in Figure 10 , as well as the global trend in the leeward side $\left(\right.$ after $90^{\circ}$ ). Nevertheless, an interesting and non expected phenomenon is observed for SSG- $\omega$ model around the $30^{\circ}$ azimuth and should be highlighted. A large peak of friction is found in this azimuth, owing to a concentration of friction due to a perturbation coming from the nose of the spheroid. This phenomenon does not correlate with the experimental results nor with the other simulations. DRSM SSG-LRR- $\omega$ seems to produce this phenomenon but in a very reduced way. In contrast, this peak does not seem to have any impact on the skin pressure or on the friction at other azimuths of the spheroid. Without any explanation found in the bibliography, this phenomenon seems to be of pure numerical origin. Furthermore, SSG- $\omega$ seems to have problems determining wall frictions in the flow separation area, showing a large amount of fluctuations. For all these reasons SSG-LRR- $\omega$ has been chosen as the DRSM model for performing the comparisons with the other simulations. Additionally, from now on the SSG-LRR- $\omega$ model will be designated as DRSM.
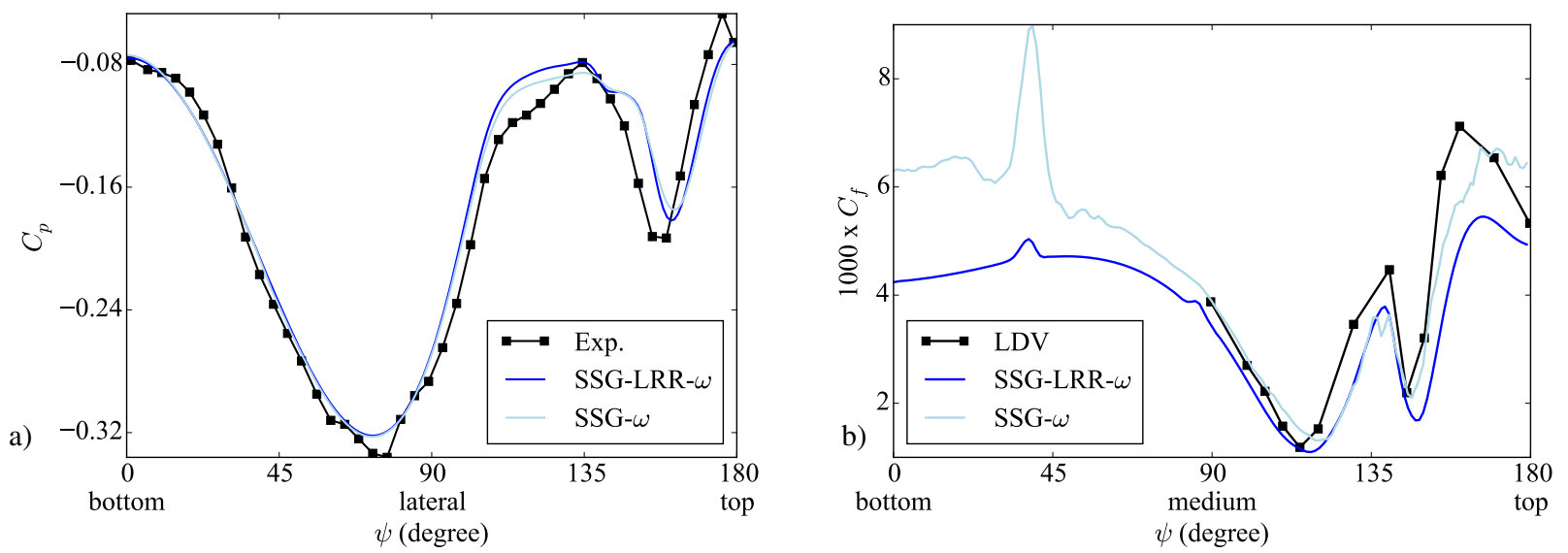

Fig. 10 Azimuthal distributions of DRSM models at a $77 \%$ longitudinal slice $20^{\circ}$ of angle of attack. a) Skin pressure coefficient and b) friction coefficient azimuthal distributions. 


\section{B. Comparison of models}

Once the comparison between several variants of a given model is completed, a comparison between the different selected models is presented in this chapter. The performance of these models is studied for the $10^{\circ}$ and $20^{\circ}$ test cases, and a deeper insight is given into their differences in order to have a better understanding of the models characteristics.
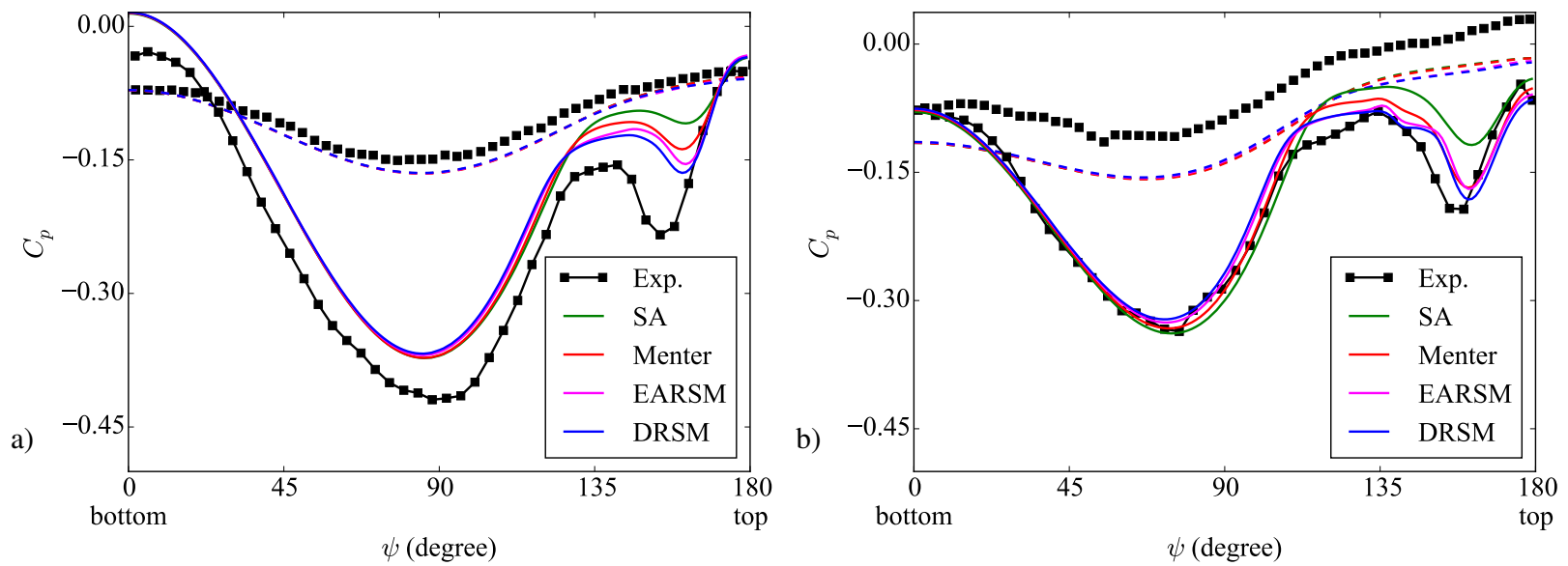

Fig. 11 Comparisons between the azimuthal distributions of skin pressure at the two stages: a) at $60 \%$ and b) at $77 \%$. In solid line the $20^{\circ}$ test cases and in dashed line the $10^{\circ}$ test cases.
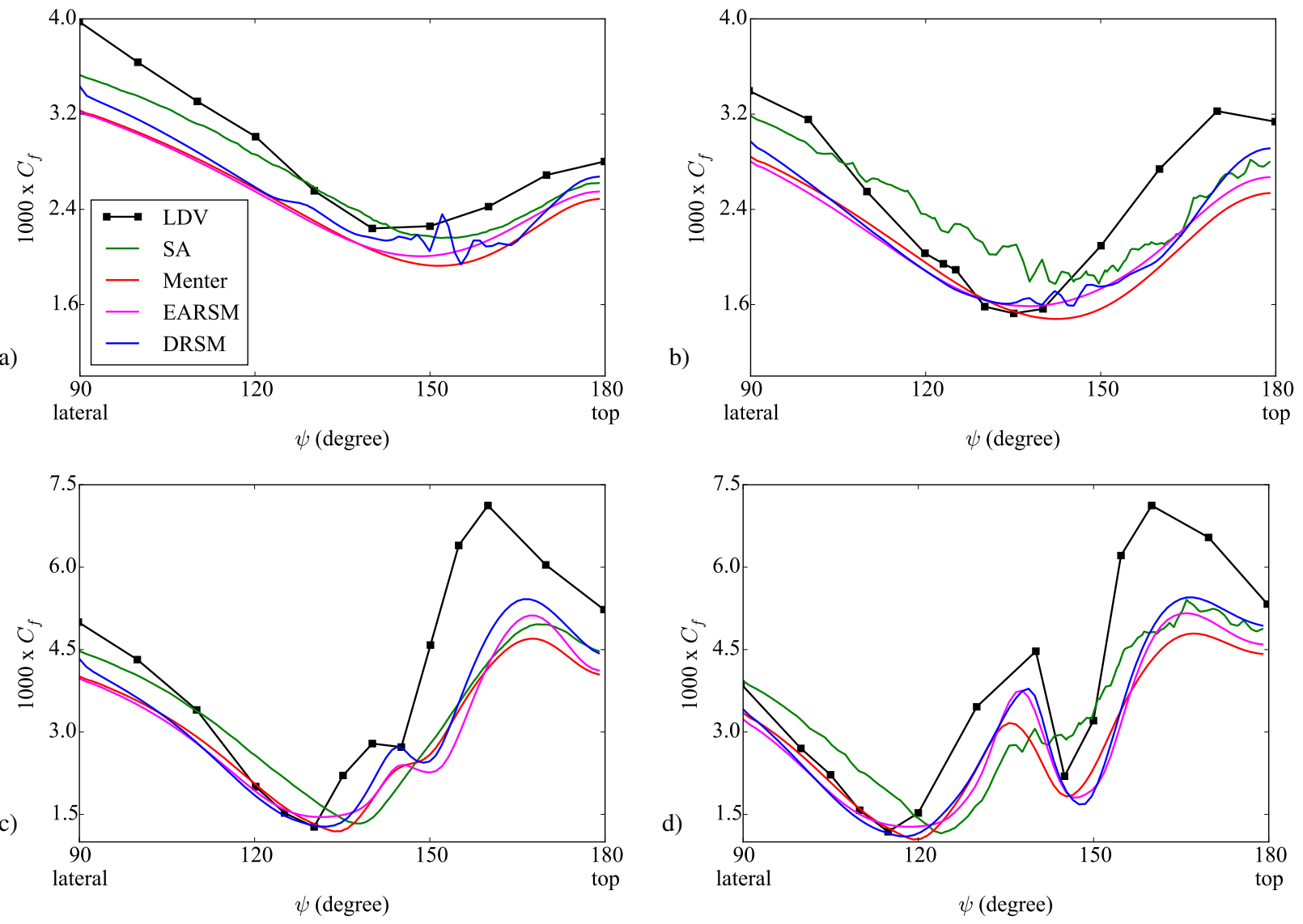

Fig. 12 Comparisons between the azimuthal distributions of the skin friction: a) and b) correspond to the $10^{\circ}$ results and c) and d) the $20^{\circ}$ ones. On the other hand, a) and c) are at the $60 \%$ slice, whilst b) and d) are at the $77 \%$ slice. 
Figure 11 shows the skin pressure coefficient azimuthal distributions at $56 \%$ and $77 \%$ longitudinal positions for the $10^{\circ}$ and $20^{\circ}$ cases. All the different models succeed to simulate the pressure distribution for the slice at $56 \%$ and $10^{\circ}$ of incidence, where no clear primary separation still exists. However, later at the $77 \%$ slice, even if all the different models predict a good trend of the pressure distribution, they underestimate it. For the $20^{\circ}$ test case, all the models underestimate the pressure distribution at 56\% length, due to the fact that flow separation is young (pressure drop between $145^{\circ}$ and $165^{\circ}$ ). Later on, at the $77 \%$ position, once the primary vortex is well established, the pressure distributions are better simulated and trends are better for most of the turbulence models. However, for this case, differences between models are found around the zone of influence of this primary vortex and at the occurrence of a secondary vortex (between $120^{\circ}$ and $160^{\circ}$ ): Menter, EARSM and DRSM models better capture the pressure maximum and minimum as well as the gradients, whilst SA model is far from the experimental measurements.

Azimuthal distributions of the friction coefficient are compared in Figure 12. The $10^{\circ}$ test case shows significant differences between the CFD models and the LDV measurements. Friction minima are slightly shifted, less than $10^{\circ}$ for EARSM and DRSM, but more for SA and Menter. In addition, friction seems to be globally underestimated. DRSM model shows fluctuations around $150^{\circ}$, which seems to be of pure numerical reasons, similar to the friction peaks of the SSG- $\omega$ described before. However, trends are globally well predicted. When studying the $20^{\circ}$ test case, larger differences between the models results are obtained: DRSM model exhibits a better capture of the friction distribution trend, especially at the $60 \%$ slice. The EARSM model also shows acceptable results in both longitudinal slices, but with a slight underestimation of the minimum around the $140^{\circ}$ azimuth, generated by the secondary vortex formation. The Menter model underestimates the friction gradients between the $130^{\circ}$ and $150^{\circ}$ azimuths; indeed, the Menter model captures the secondary vortex more downstream, provoking this underestimation. The SA model does not succeed in capturing the secondary vortex and thus this model does not correctly simulate the friction gradients in this region. Nevertheless, no model succeed to well predict the minima and maxima positions and intensities of skin-friction variations created by the primary and the secondary vortex with precision. This will have a direct impact on the shape and positions of the velocity profiles in the boundary layer that will be compared later in this study.

From friction calculations, and using the crossflow friction criterion presented in Section III.C. the separation locations are calculated and compared with the experimental measurements obtained by oil flow visualisations for the two test cases (Figure 13). Separation lines are a reflection of the azimuthal distribution of the skin-friction coefficient discussed above. Indeed, as the prediction of the skin-friction distribution is most of the time qualitatively correct, the location of the primary and secondary flow separations are also generally well predicted, with only small differences at some specific locations. As a matter of fact, CFD models tend to capture primary flow separation more upstream than in the experiment. This is probably due to the difficulties of determining the first separation points from flow visualizations. Indeed, the vortex generated by flow separation needs to be sufficiently developed in order to get a friction line pattern clear enough to detect flow separation. EARSM and DRSM succeed in capturing the secondary separation better than the other two models, as it was shown when discussing the the friction distributions of Figure 12 On the other hand, SA model captures secondary separation too downstream, showing that this model does not seem well adapted for capturing this type of flows. Likewise, DRSM model shows discrepancies with the other models at the beginning of the primary separation for the $10^{\circ}$ test case: indeed as shown in the previous chapter, DRSM models have difficulties in predicting skin-friction accurately, certainly due to numerical issues as turbulent dissipation is significantly reduced with respect to first-order models. Nevertheless, this issue seems to have no impact further downstream when vortices are well established.

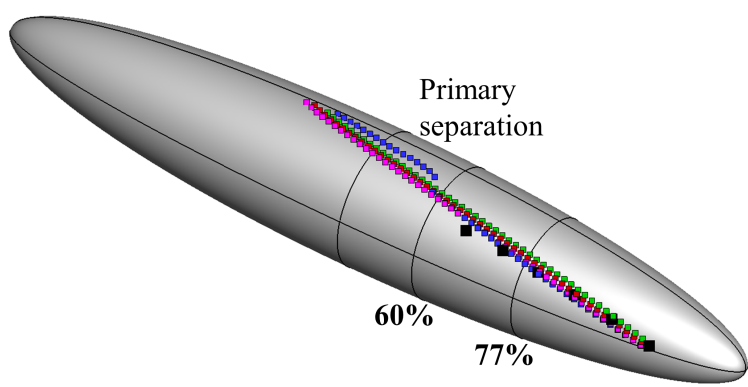

a)

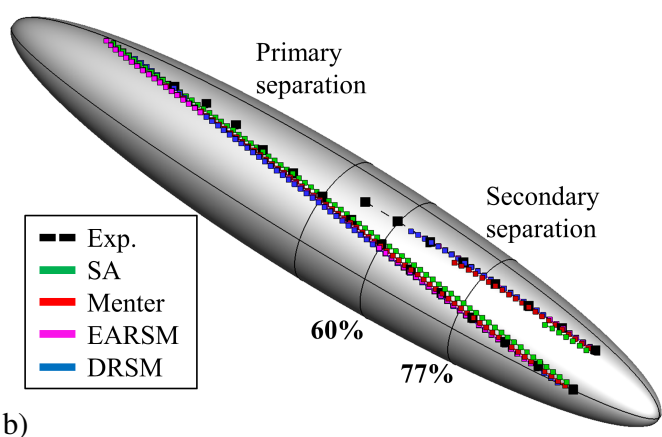

b)

Fig. 13 Visualisation of the experimental and calculated separation lines: a) $10^{\circ}$ of incidence test case, b) $20^{\circ}$ of incidence test case. 
Experimental velocity profiles are compared with the CFD calculations at 77\% length for the two angle of attack test cases $\left(10^{\circ}\right.$ results in Figure 14 and $20^{\circ}$ in Figure 16). Results are shown in a Body Surface Coordinate System (Figure 2), with $U$ tangential to the body surface and pointing to the tail, $V$ normal to the surface, and $W$ tangential to the surface and forming a right-handed trihedral. For clarity purposes, only the $77 \%$ longitudinal slices and the profiles at azimuths $120^{\circ}, 145^{\circ}$ and $160^{\circ}$ are shown in this paper. Nevertheless, the $60 \%$ slices and all others different azimuths either do not provide additional information or lead to the same conclusions.
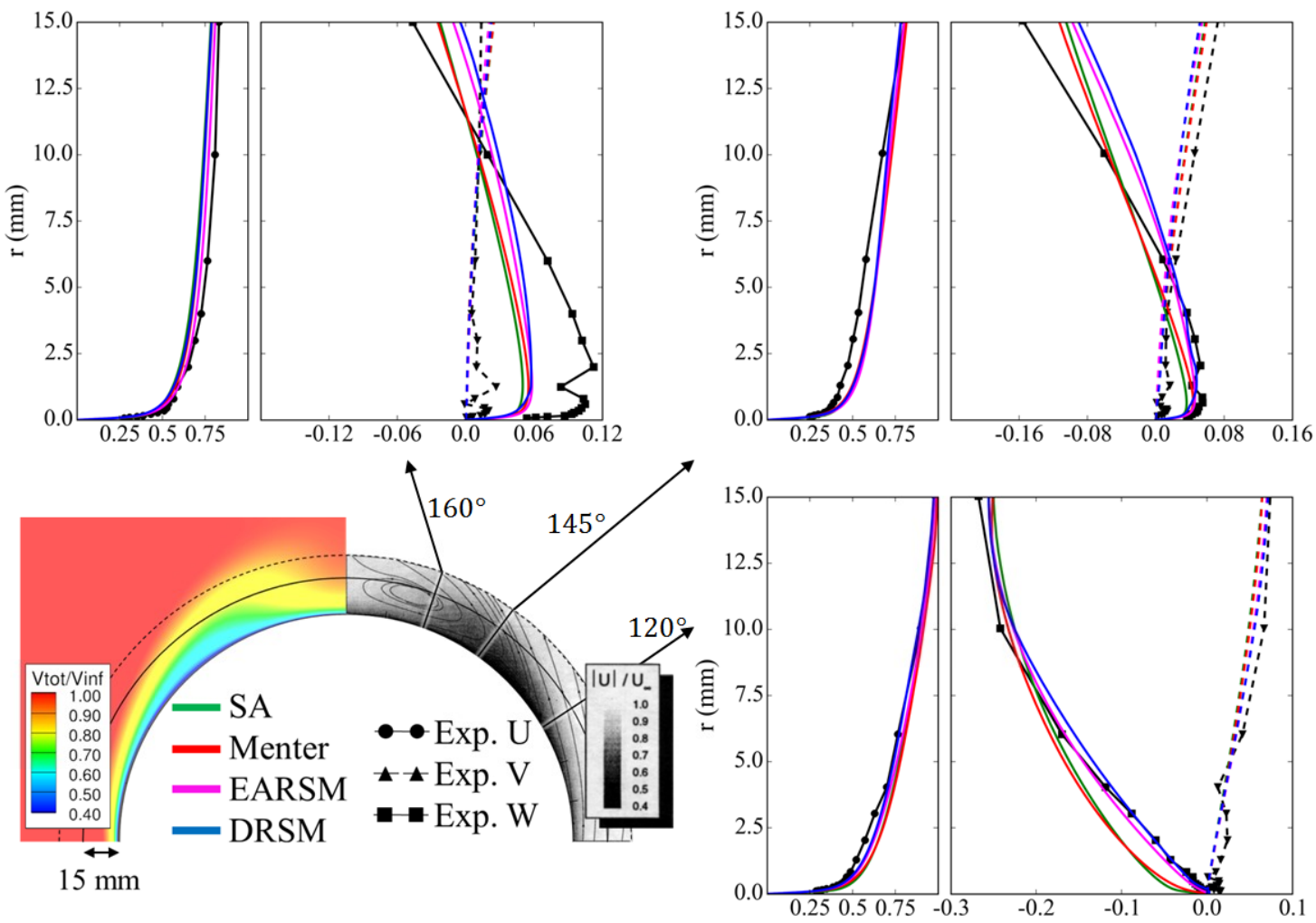

Fig. 14 Comparison between experimental and simulated velocity profiles for several azimuths at the $\mathbf{7 7 \%}$ longitudinal position of the $10^{\circ}$ test case at a body surface coordinate system. For each azimuth, the left curve represents $U / U_{\infty}$, and at the right one the cross velocities: $V / U_{\infty}$ (dashed lines and $\boldsymbol{\nabla}$ ), and $W / U_{\infty}$ (solid lines and $\square)$. Lower left figure shows the contours of velocity from experiments and EARSM simulations. LDV limit in dashed line; in solid line the velocity profiles radial limit (15 $\mathrm{mm}$ from the surface).

a)

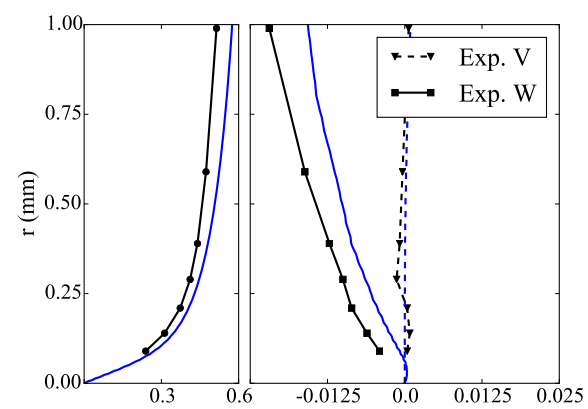

b)

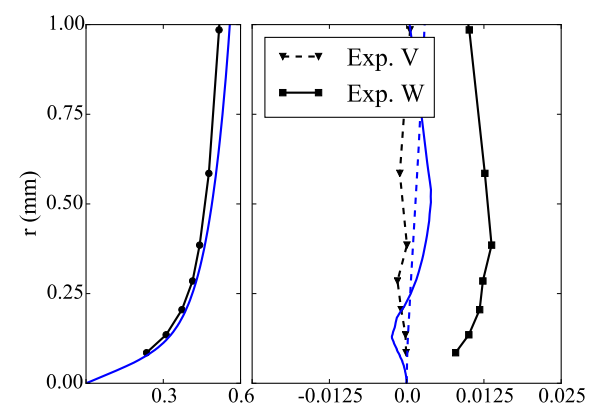

Fig. 15 Zoom at the closest part the wall of the velocity profiles for a) $145^{\circ}$ and b) $150^{\circ}$ azimuths at the $60 \%$ longitudinal position of the $10^{\circ}$ test case for DRSM calculations. For each azimuth, the left figure represents $U / U_{\infty}$, and at the right one are the cross velocities: $V / U_{\infty}$ as $\mathbf{v}$ and dashed lines, and $W / U_{\infty}$ as $\square$ and solid lines. 
Numerical results obtained for the $10^{\circ}$ incidence test case show a good agreement with the experimental measurements (Figure 14). Velocity profiles in the boundary layer are rather well captured by all the models, with only slight differences with experiments. This differences are greater when studying the near wall parts of the profiles. Similar behaviours are found between SA and Menter models, and EARSM and DRSM models, the latter models being generally more accurate. However, as shown in Figure 15, a strange and probably non-physical behaviour is found for the DRSM model at the closest parts of the velocity profiles (until $\sim 0.25 \mathrm{~mm}$ from the surface) between $145^{\circ}$ and $155^{\circ}$. In this figure, the velocity profiles at $145^{\circ}$ and $150^{\circ}$ azimuths of the $60 \%$ position are presented, showing that the $W$ velocity components are shifted towards lower magnitudes from the experimental ones in this zone. Indeed, they present a significant shape change from the $145^{\circ}$ azimuth to the $150^{\circ}$ one under the influence of the primary vortex. The $W$ velocities calculated by the DRSM model near the wall present large unexpected gradients, which explains the fluctuations found in the azimuthal friction distributions (Figure 12 a). Possibly, an additional and small vortex is predicted inside the primary one.

Velocity profiles of the $20^{\circ}$ are presented in Figure 16). As in the previous case, differences between models at the $77 \%$ longitudinal slice are more significant than at $60 \%$ (not shown here). This differences are especially large between the $130^{\circ}$ and $160^{\circ}$ azimuths where the primary separation and the secondary vortex are found. This case shows that the velocity $W$ (perpendicular to the spheroid axis and tangential to the surface) is the most difficult velocity component to predict for all models. Indeed, significant velocity gradients are obtained, which strongly depend on the position and intensity of the primary and secondary vortex. This velocity component is generally underestimated close to the wall where the largest gradients are found. Generally speaking, EARSM and DRSM models provide fairly satisfactory predictions of the velocity components. Both models indeed show velocity profiles close to the experimental measurements, even in the vicinity of the secondary vortex. On the other hand, Menter model yields fairly satisfactory predictions of the primary vortex influence, but the effect of the secondary vortex is poorly predicted. In addition, all the models underestimate the $W$ velocity component between the azimuths $160^{\circ}$ and $170^{\circ}$. This underestimation is translated into the underestimation of the friction maximum observed in Figure 12 in the same azimuth range.
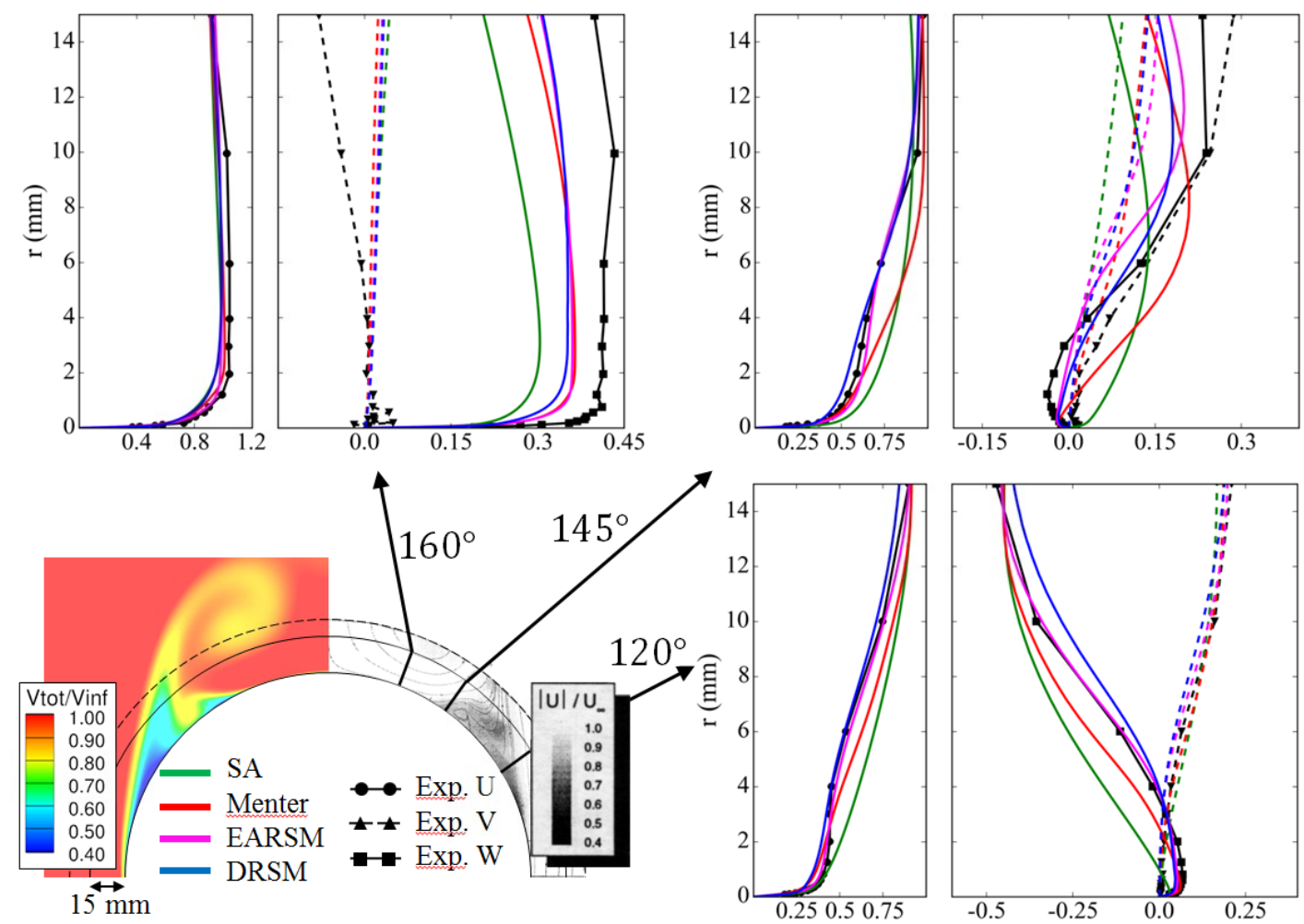

Fig. 16 Comparison between experimental and simulated velocity profiles for several azimuths at the $77 \%$ longitudinal position of the $20^{\circ}$ test case at a body surface coordinate system. For each azimuth, the left curve represents $U / U_{\infty}$, and at the right one the cross velocities: $V / U_{\infty}$ (dashed lines and $\mathbf{v}$ ), and $W / U_{\infty}$ (solid lines and $\square$ ). Lower left figure shows the contours of velocity from experiments and EARSM simulations. LDV limit in dashed line; in solid line the velocity profiles radial limit ( $15 \mathrm{~mm}$ from the surface). 
As the velocities induced by flow separation strongly depend on the position of the primary and secondary vortices, it is interesting to compare experimental velocity profiles with simulated results in a range of azimuths surrounding the experimental position (e.g. \pm few degrees around the experimental azimuth). These type of comparisons are shown in Figure 17, for the azimuths $120^{\circ}, 140^{\circ}$ and $150^{\circ}$ at the $77 \%$ slice of the $20^{\circ}$ test case. As SA model does capture secondary separation too far downstream along the spheroid, any close velocity profile fails in predicting the $W$ velocity at $140^{\circ}$. Indeed, the $77 \%$ experimental position should be compared with a more downstream one computed with SA model, where the secondary separation would be completely established. This same issue happens with Menter model in a much lesser extend, thus allowing this model to provide better correlations with experiment than SA. EARSM and DRSM models globally succeed in reproducing the experimental gradient in a range of $\pm 5^{\circ}$ for the three velocity components and the three azimuths shown. Indeed, although the predicted $\mathrm{W}$ velocity component at $140^{\circ}$ azimuth is shifted from measured data,both have similar velocity gradients.
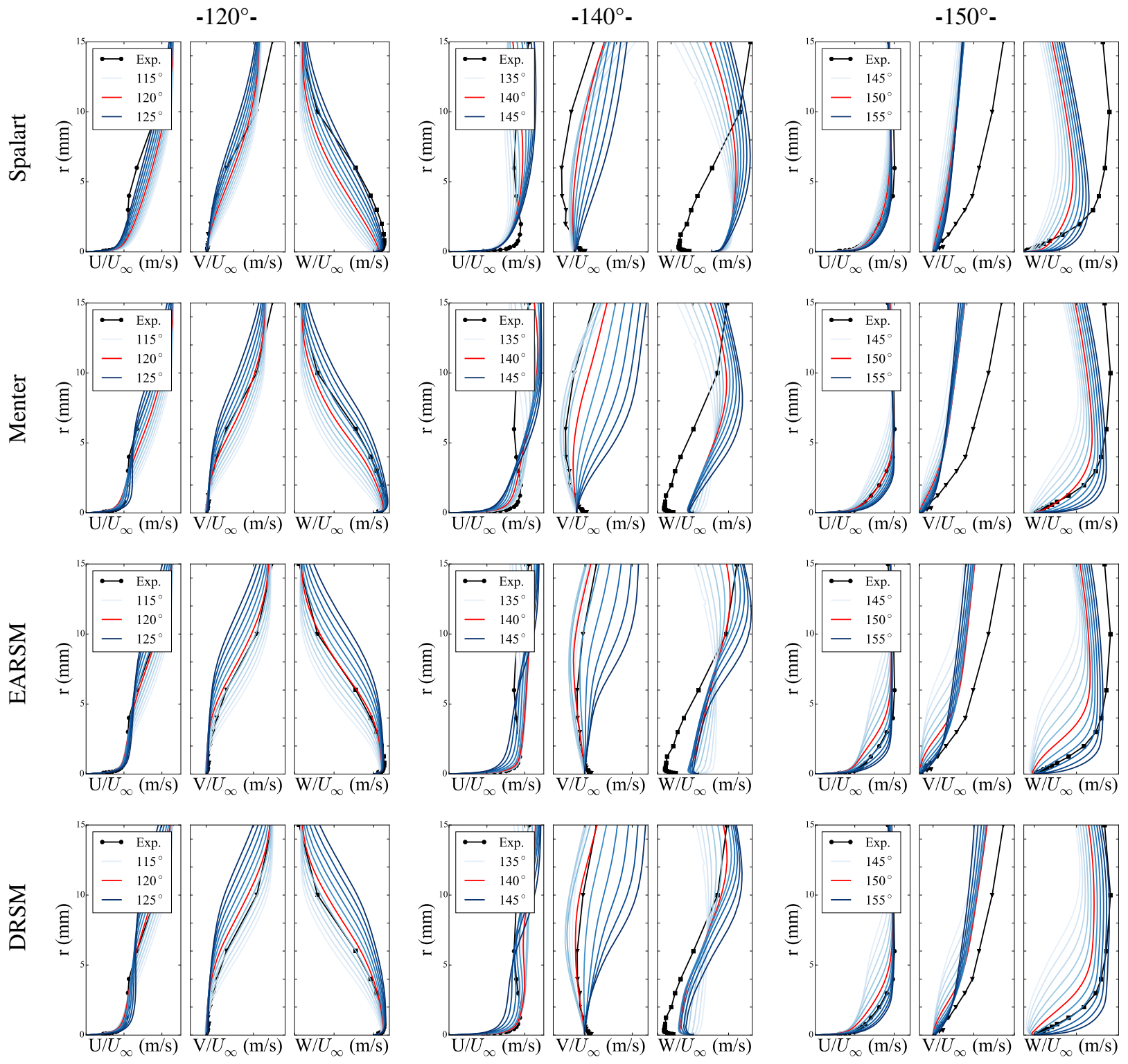

Fig. 17 Comparison between boundary layer velocity profiles from experiments and the four models, in a Body Axis Coordinate System. Left figures $120^{\circ}$, middle figures $145^{\circ}$ and right figures $160^{\circ}$. Velocities in a Body Surface coordinate system. 
Unlike SA, Menter or EARSM, DRSM model provides the Reynolds stress tensor, which can be compared to the experimental data. These Reynolds stress are shown in Figure 18 , Even if some Reynolds stress profiles follow the experimental trends, generally poor correlations with the experiments are obtained. With the exception of $\langle w w>$, normal Reynolds stresses trends seem to be better captured than shear ones. However, as was the case with velocity profiles, sheared regions gather the most complex phenomena, and thus Reynolds stress in these regions are the most difficult to simulate.
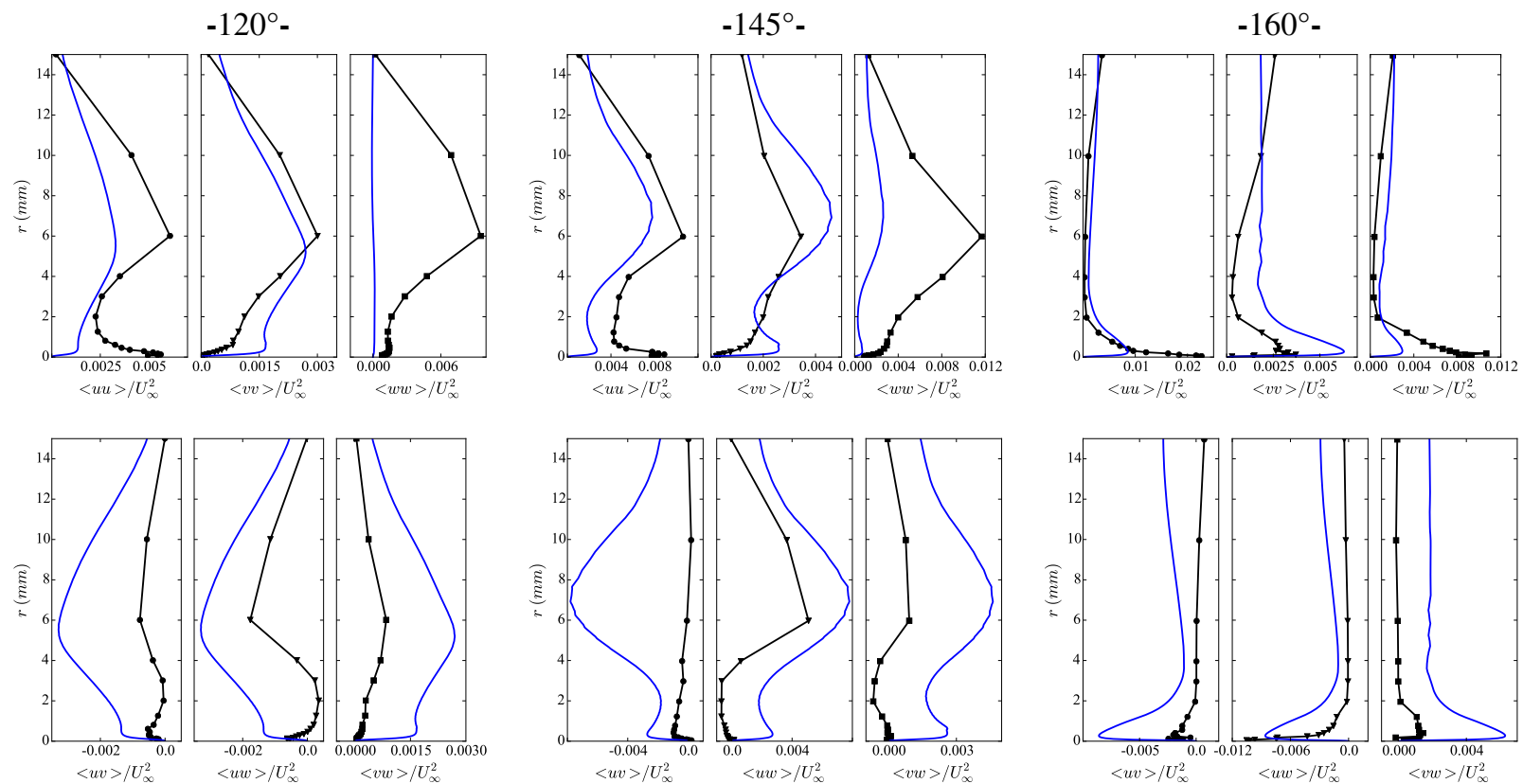

Fig. 18 Comparison between non-dimensional Reynolds Stress profiles from experiments and DRSM results, in a Body Axis Coordinate System. Figures above are the normal stress $(<u u\rangle,<v v>$ and $<w w>$ ), Figures below are the shear stress $(<u v\rangle,<u w>$ and $<v w>)$. Left figures $120^{\circ}$, middle figures $145^{\circ}$ and right figures $160^{\circ}$.

\section{Comparison of convergence rates}

Finally, from an end-user perspective it is also interesting to compare the convergence rate of the different simulations. In Figure 19 lift convergence is plotted against time. Computational time is expressed as CPU time. In this figure, the convergence point is marked with a dot for each simulation. This point is estimated using a linear regression of the lift evolution, and taking the point where the slope of the curve is equal to $1.5 \times 10^{-7}$ (and therefore negligible).

During this study, convergence of lift and drag forces as well as pitching moment were studied. Here, only the lift is presented because it was the most challenging force to converge. SA model shows the fastest convergence of lift, followed by EARSM and Menter, whilst DRSM is the slowest model to converge. Indeed, the number of equations to consider during the simulations directly affect the convergence rate. SA only solves one equation for the turbulent viscosity, whereas DRSM solves for the six Reynolds stress components and $\omega$. DRSM models are also less dissipative, this reducing the convergence rate of the simulations. On the other hand, EARSM and Menter simulations show a very similar convergence time. No exploitable data of the experimental lift were found, thus no qualitative comparisons are possible. No fluctuations were found on this component or on the other ones (drag, pitch moment, ...), which explains why URANS simulations seemed unnecessary. 


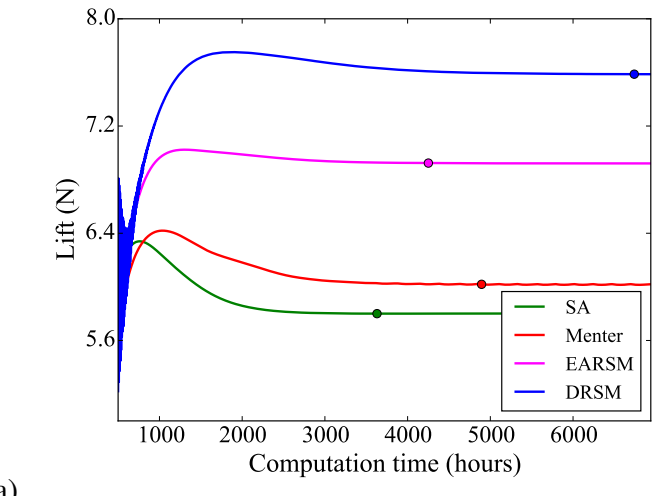

a)

Fig. 19 Computations convergence time in hours. The convergence point is indicated as a $\circ$. a) $10^{\circ}$ of incidence test case, b) $20^{\circ}$ of incidence test case.

\section{Conclusion and outlook}

This study focuses on analyzing crossflow separation physics and developing a methodology for capturing the phenomenon by numerical means. A 6:1 prolate spheroid at incidences of $10^{\circ}$ and $20^{\circ}$ was used as test cases for comparing simulations with the experimental data. These test cases present a complex flow structure with one or two crossflow separations giving rise to one or two pairs of counter-rotating vortices, depending on the angle of attack considered $\left(10^{\circ}\right.$ or $20^{\circ}$ respectively). Very different RANS models were compared, with emphasis put on four of them: Spalart-Allmaras, k- $\omega$ Menter SST, Hellsten k- $\omega$ EARSM and SSG-LRR- $\omega$ DRSM. Comparisons of skin-pressure and friction azimuthal distributions, as well as boundary layer velocities were accomplished for assessing the models' accuracy. In addition, the crossflow separation position were determined using a simple criterion from the literature. Neither the wind tunnel walls or the transition trip were considered during simulations. Indeed, farfield boundary conditions and fully turbulent computations were considered in the present work. Nevertheless, overall good results are found.

The Menter, EARSM and DRSM models provide fairly good correlations with the experimental measurements. More specifically, EARSM and DRSM seem to be the most accurate when considering skin pressure and friction distributions. Maxima and minima values are fairly well positioned, even if the beginning of the secondary separation is difficult to capture as the flow is strongly three-dimensional. In contrast, SA model has difficulties to capture secondary separation, detecting it too far downstream. This behaviour has been previously found in the literature and it is probably due to its large turbulent viscosity in the viscous layers. When considering the boundary layer velocity profiles, again EARSM and DRSM models are the most accurate ones, showing overall good behaviour, and they are followed by Menter, and finally SA. However, all the models have difficulties simulating the near wall part of the boundary layer in the secondary separation zone, where the most pronounced gradients are found.

Considering the present results and the speed of convergence of the different models, EARSM seems to offer the best compromise between accuracy and convergence time from the present comparison of models. On the other hand, SSG-LRR- $\omega$ DRSM model presents also very good correlations with the experiments, even if its speed of convergence is less interesting and if it generates non physical fluctuations of the skin-friction. Therefore, these models are now planned to be tested on a real helicopter geometry to assess their performance on a more complex configuration which induces crossflow separation, as well as many other flow phenomena.

\section{Aknowledgments}

This work was supported by the CHAllenge AeRo-thermo-MEcanique (CHARME) Project, partially funded by Direction Générale de l'Aviation Civile (DGAC), and by Association Nationale de la Recherche et de la Technologie (ANRT). The authors are grateful for the support from Luis Bernardos in the separation study and Sébastien Deck in the model selection. 


\section{References}

[1] Roesch, P., and Dequin, A., "Experimental Research on Helicopter Fuselage and Rotor Hub Wake Turbulence," Journal of the American Helicopter Society, Vol. 30, No. 1, 1985, pp. 43-51. doi:doi:10.4050/JAHS.30.1.43.

[2] Mazzucchelli, C., and Wilson, F., "The Achievement of Aerodynamic Goals on the EH101 Project through the 'Single Site'Concept," 1991.

[3] Wetzel, T., Simpson, R., and Chesnakas, C., "Measurement of three-dimensional crossflow separation," AIAA Journal, Vol. 36, 1998, pp. 557-564. doi:10.2514/3.13861.

[4] Ahn, S., and Simpson, R., “An experimental study of flow over a 6 to 1 prolate spheroid at incidence,” Ph.D. thesis, 051992.

[5] Chesnakas, C., and Simpson, R., "Full three-dimensional measurements of the cross-flow separation region of a 6:1 prolate spheroid," Experiments in Fluids, Vol. 17, No. 1, 1994, pp. 68-74. doi:10.1007/BF02412805, URL https: //doi.org/10.1007/BF02412805

[6] Chesnakas, C., and Simpson, R., "Measurements of the Turbulence Structure in the Vicinity of a 3-D Separation," Journal of Fluids Engineering-transactions of The Asme - J FLUID ENG, Vol. 118, 1996. doi:10.1115/1.2817373.

[7] Wetzel, T., and Simpson, R., "Unsteady Flow Over a 6:1 Prolate Spheroid," Rept. api-aoe-232, blacksburg, va, Dept. of Aerospace and Ocean Engineering, Virginia Polytechnic Inst. and State Univ., 041996.

[8] Goody, M., and Simpson, R., "An Experimental Investigation of Pressure Fluctuations in Three-Dimensional Turbulent Boundary Layers," 1999, p. 337.

[9] Tsai, C.-Y., and Whitney, A., "Numerical Study of Three-Dimensional Flow Separation for a 6:1 Ellipsoid," 37th AIAA Aerospace Sciences Meeting and Exhibit, 1999.

[10] Rhee, S., and Hino, T., "Computational Investigation of 3D Turbulent Flow Separation around a Spheroid using an Unstructured Grid Method," Journal of the Society of Naval Architects of Japan, Vol. 2000, No. 188, 2000, pp. 1-9.

[11] Wikström, N., U., S., N., A., and C., F., "Large eddy simulation of the flow around an inclined prolate spheroid," Journal of Turbulence, Vol. 5, 2004, p. N29. doi:10.1088/1468-5248/5/1/029.

[12] Constantinescu, G., Pasinato, H., Wang, Y., and Squires, K., "Numerical investigation of flow past a prolate spheroid," 40th AIAA Aerospace Sciences Meeting and Exhibit, 2002.

[13] Fureby, C., Alin, N., Wikström, N., Menon, S., Svanstedt, N., and Persson, L., "Large eddy simulation of high-Reynolds-number wall bounded flows," AIAA journal, Vol. 42, No. 3, 2004, pp. 457-468.

[14] Xiao, Z., Zhang, Y., Huang, J., Chen, H., and Fu, S., "Prediction of separation flows around a 6:1 prolate spheroid using RANS/LES hybrid approaches," Acta Mechanica Sinica, Vol. 23, No. 4, 2007, pp. 369-382.

[15] Mor-Yossef, Y., Separated Flow Prediction Around a 6:1 Prolate Spheroid Using Reynolds Stress Models, Springer International Publishing, Cham, 2015, pp. 39-60.

[16] Fureby, C., and Norrison, D., "RANS, DES and LES of the Flow Past the 6:1 Prolate Spheroid at ang10 and ang20 Angle of Incidence," AIAA Scitech 2019 Forum, 2019. doi:10.2514/6.2019-0085.

[17] Cambier, L., Heib, S., and Plot, S., “The Onera elsA CFD software: input from research and feedback from industry,” Mechanics \& Industry, Vol. 14, 2013, p. 159-174. doi:https://doi.org/10.1051/meca/2013056.

[18] Liou, M.-S., and Edwards, J., "AUSM schemes and extensions for low Mach and multiphase flows," 30 th CFD Von Karman Lecture Series, 1999.

[19] Spalart, P., and Allmaras, S., “A One-Equation Turbulence Model for Aerodynamic Flows,” AIAA, Vol. 439, 1992. doi: 10.2514/6.1992-439.

[20] Menter, F., “Two-equation eddy-viscosity turbulence models for engineering applications,” AIA Journal, Vol. Vol. 32, No. No. 8, 1994, pp. 1598-1605. doi:10.2514/3.12149.

[21] Kok, J., "Improvements of Two-Equation Turbulence Models in Multi-Block Flow Solvers: Free-Stream Dependency and Transition,” Tech. rep., AVTAC/TR/NLR/JCK990520/Draft2, 1999. 
[22] Spalart, P., "Strategies for Turbulence Modelling and Simulations," International Journal of Heat and Fluid Flow, Vol. Vol.21, No. 2000, 2000, pp. pp. 252-263.

[23] Hellsten, A., "New two-equation turbulence model for aerodynamics applications," Phd dissertation, Helsinki University of Technology. Laboratory of Aerodynamics, 022004.

[24] Aupoix, B., "Improving the coupling of the Speziale, Sarkar and Gatski differential Reynolds stress model with an $\omega$-scale equation,” Rt 1/13929, 2012.

[25] Cécora, R., Radespiel, R., Eisfeld, B., and Probst, A., "Differential Reynolds-Stress Modeling for Aeronautics," AIAA Journal, Vol. 53, No. 3, 2014, pp. 739-755.

[26] Meier, H. U., and Kreplin, H. P., "Experimental Investigation of the Boundary Layer Transition and Separation on a Body of Revolution,” Z. Flugwiss. Weltraumforschung, Vol. Vol. 4, No. No. 2, 1980, pp. pp. 65-71.

[27] Delery, J., "Toward the Elucidation of Three-Dimensional Separation," Annual Review of Fluid Mechanics, Vol. 33, 2001, pp. 129-154. URL https://ci.nii.ac.jp/naid/10024679825/en/

[28] Han, T., and Patel, V., "Flow separation on a spheroid at incidence," Journal of Fluid Mechanics, Vol. 92, No. 4, 1979, p. 643-657. doi:10.1017/S002211207900080X.

[29] Kreplin, H. P., Vollmers, H., and Meier, H. U., "Shear Stress Measurements on an Inclined Prolate Spheroid in the DFVLR 3 m x 3 m Low Speed Wind Tunnel, Göttingen-Data Report,” Tech. rep., 011985. 